\title{
Adherence to best practice consensus guidelines for familial Mediterranean fever: a modified Delphi study among paediatric rheumatologists in Turkey
}

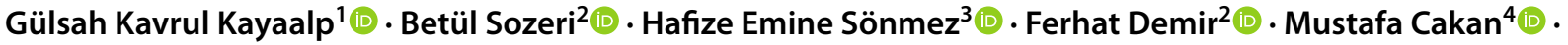

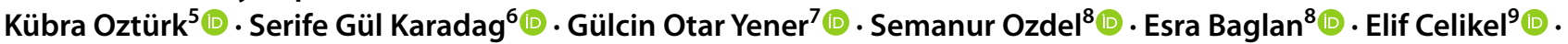

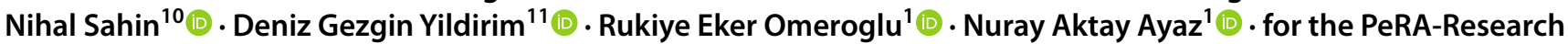 \\ Group
}

Received: 30 October 2020 / Accepted: 23 December 2020 / Published online: 16 January 2021

(c) The Author(s), under exclusive licence to Springer-Verlag GmbH, DE part of Springer Nature 2021

\begin{abstract}
Background Although not validated fully, recommendations are present for diagnosis, screening and treatment modalities of patients with familial Mediterranean fever (FMF).

Objective To review the current practices of clinicians regarding FMF and reveal their adherence to consensus guidelines. Methods Fifteen key points selected regarding the diagnosis and management of FMF were assessed by 14 paediatric rheumatologists with a three-round modified Delphi panel.

Results Consensus was reached on the following aspects: genetic analysis should be ordered to all patients when clinical findings support FMF, but its result is not decisive alone. In the absence of clinical features, colchicine should be commenced when two pathogenic alleles and family history of amyloidosis are present. Serum amyloid A testing at each visit is recommended in patients resistant to colchicine, with subclinical inflammation and family history of amyloidosis. Consensus was reached on both the definition of colchicine resistance and starting biologic in resistant cases. Cost, efficiency, ease of use, treatment adherence, accessibility and emergence of adverse events are the factors affecting the choice of biologic agents. In patients without any attack and evidence of subclinical inflammation within the last 6 months following initiation of biologics, treatment dose intervals can be prolonged.

Conclusion A consensus was achieved regarding the routine diagnosis and screening and treatment of FMF patients. The definition of colchicine resistance was made and a protocol was created for prolongation of treatment intervals of biologic agents. We anticipate that the results of the study reveal real-life data on the approach to patients in clinical practice.
\end{abstract}

Keywords Familial Mediterranean fever $\cdot$ Children $\cdot$ Delphi technique $\cdot$ Colchicine $\cdot$ Colchicine resistance $\cdot$ Biological agents

\section{Introduction}

Familial Mediterranean fever (FMF) is the most prevalent inherited autoinflammatory disease and is considerably common among people from the Mediterranean basin such as

Supplementary Information The online version contains supplementary material available at https://doi.org/10.1007/s0029 6-020-04776-1.

Nuray Aktay Ayaz

nurayaktay@gmail.com

Extended author information available on the last page of the article
Turks, Arabs, non-Ashkenazi Jews and Armenians, but is increasingly being reported from other countries not related to this region [1]. It is characterized by recurrent inflammatory attacks of fever and serositis, and increased risk of amyloidosis [2]. Colchicine is the standard treatment and is endorsed effective in preventing attacks and development of amyloidosis [3]. Biologic treatments such as interleukin-1 (IL-1) blocking agents are considered as a treatment of choice for colchicine resistant patients [3-5].

Highly variable phenotypes exist among FMF patients due to the differences in gene penetration, the impact of environmental factors and presence of modifier genes influencing the development of amyloidosis [6]. The heterogeneous 
course of disease complicates the diagnostic and therapeutic approaches of the physicians. Two sets of diagnostic criteria, the classic Tel Hashomer criteria and more recent Livneh criteria, have been developed for the diagnosis of FMF in adult patients to provide convenience and standardization in diagnosis [7]. In 2009 a new criteria for paediatric patients was put forward by a Turkish group [8], and validated in French children and in different ethnic groups in Europe and eastern Mediterranean basin $[9,10]$. Most recently, the new Eurofever/Paediatric Rheumatology International Trials Organisation (PRINTO) criteria was introduced in 2019, which combined clinical manifestations with genotype for the first time [11] (Supplementary document). Although there are diagnostic criteria, diagnosis of FMF depends mainly on clinical basis and on the level of experience of the clinician, and there are some conflicting issues such as atypical clinical findings with the presence of a positive genetic test [12]. Furthermore, response to treatment with colchicine or IL-1 blocking agents differ among patients and there is still no consensus for the definition of inadequate response to colchicine for switching the treatment to IL-1 blocking agents [13].

In 2015, recommendations for genetic diagnosis of FMF were introduced by "Single Hub and Access point for paediatric Rheumatology in Europe" (SHARE). The following year, "European League Against Rheumatism" (EULAR) introduced the recommendations for diagnosis and management of FMF $[12,14]$. Turkey is one of the countries with the highest prevalence of FMF estimated as 1/1000 [1, 15], so it's of interest both to document the clinical practices of the experienced paediatric rheumatologists dealing with a huge patient population and their adoption to existing formal recommendations and current guidelines, as real-life data. A consensus based on the real-life data obtained from an FMF prevalent country might be a model for the clinicians dealing with the diagnosis and management of FMF. This study aims to reveal adherence of clinicians to existing guidelines and document the current clinical practices of field experts from tertiary centres in Turkey, regarding the diagnosis and management of FMF. Another objective of the study is to settle a consensus based protocol for prolongation of biologic treatment dose intervals when patients are accepted to be in remission.

\section{Methods}

\section{Study participants}

The study was conducted by the Paediatric Rheumatology Academy-Research Group (PeRA-RG). The members of the PeRA-RG are paediatric rheumatologists (PRs) working in regional tertiary centres in different cities of Turkey
[16]. The invitation mails for the study were sent to 20 PRs who are members of PeRA-RG. Fourteen PRs accepted to participate in the study and six PRs denied to participate either due to their busy working schedule or due to COVID19 pandemics. The Delphi panel consisting of 14 panellists from 11 centres was established. All participants are expert in diagnosis, follow-up and management of FMF and number of FMF patients seen per week by each PRs was at least 40. (Supplementary document).

\section{Delphi method}

Delphi is a method for structuring a group communication process to deal with a complex problem [17]. It is conducted via multi-step questionnaires preserving participant anonymity, and by providing feedback information to participants between steps. It has been widely used across many disciplines including rheumatology, to elicit consensus on a topic, but it is also used to investigate a wide range of opinions without achieving a consensus. Since the Delphi technique does not require face-to-face meetings, it allows group communication between people in different geographic areas $[18,19]$.

In the first stage of the Delphi exercise of this study, a detailed literature search was performed by a paediatric rheumatology fellow (GKK) and a review of the literature was carried out and a shortlist of $15 \mathrm{key}$ points were selected by three paediatric rheumatologists (NAA, BS, HES). These key points comprised of; (1) clinical and laboratory parameters for diagnosis and initiation of colchicine therapy, (2) requesting genetic analysis, (3) the impact of the result of genetic analysis on commencing colchicine treatment, (4) follow-up frequency of patients, (5) monitorization of the patients by laboratory parameters during follow-up, (6) evaluation of FMF attacks, (7) utilization of outcome measurements, (8) adjustment of colchicine dosage, (9) evaluation of the response to colchicine, (10) decision making for resistance to colchicine therapy, (11) indications for commencing biologic treatment, (12) factors affecting biologic drug selection, (13) evaluation of the response to biologic treatment, (14) convenience of prolongation of biologic treatment dose intervals when the patient is in remission, and (15) settling a protocol for prolongation of the intervals between biologic doses.

A three-round modified Delphi panel was assessed by all panellists by answering questionnaires sent via an e-mail. In the first round of the Delphi exercise, panellists were requested to answer open-ended questions to define their general opinions and stimulate the generation of new ideas. In the second round, key points were converted to statements with a 9-point Likert scale $(1=$ strongly disagree, $9=$ strongly agree). In the third round, the statements that could not be reached consensus in the previous round were 
asked again. A summary of the previous answers of the entire group was provided before each round and the panellists were requested to re-evaluate their answers.

\section{Ethics}

Approval was obtained for the study protocol from the Ethics Committee of Istanbul University, Istanbul Faculty of Medicine (approved: 31/08/2020-145479). The study was carried out complied with the Declaration of Helsinki.

\section{Data management and statistical analysis}

Data from each statement was collected using Microsoft Excel (Microsoft Corporation, Redmond, WA) and SPSS 17.0 (IBM, Armonk, NY) for the survey analysis. Descriptive statistics including mean, mode, median, percentage and interquartile ranges were conducted for each statement.

A statement was deemed consensual in agreement if it was voted 7-9 on the Likert scale by at least $75 \%$ of the participants, and the mean of all responses were greater than 7 for this statement. A statement was deemed consensual in disagreement if it was voted 1-3 on the Likert scale by at least $75 \%$ of the participants, and the mean of all responses were less than 3 for this statement.

\section{Results}

A total of 14 PRs from 11 paediatric rheumatology centres were involved to the study. All participants completed the survey. It was reported that 6 PRs were examining 80-120, 6 PRs were examining 40-80, 2 PRs were examining 120-160 children with the diagnosis of FMF per week. The frequency of colchicine resistance among patients they followed was asked to the panellists; 10 PRs reported the frequency as $5-10 \%, 3$ PRs as $10-14 \%$ and 1 PR as $15-20 \%$.

Turkish paediatric FMF criteria is used by all panellists, Eurofever/PRINTO criteria is used by 9 panellists, Tel Hashomer criteria is used by 7 panellists and Livneh criteria is used by 1 panellist.

\section{Consensus reached statements}

The statements that reached a consensus regarding to diagnosis of FMF, colchicine therapy, management of the patients with FMF, evaluation of response to colchicine and biologic treatment are presented in the Table 1 .

For diagnosis; a consensus was reached for the following aspects: FMF should be diagnosed according to certain classification criteria, genetic analysis should be ordered when clinical findings support FMF, the result of genetic testing alone cannot be considered decisive for diagnosis (Table 1).
For colchicine treatment; a consensus was reached for the following aspects: colchicine therapy should be initiated at the time of diagnosis, colchicine therapy should be initiated when two pathogenic alleles and family history of amyloidosis are present, colchicine therapy should be started at low doses and dose should be increased to receive a response without side effects until the maximum dose recommended for the patient's age is reached and presence of subclinical inflammation is an indication for increasing colchicine dose (Table 1).

For follow-up; a consensus was reached for the following aspects: screening periods of patients with FMF should not be longer than 6 months of duration, the newly initiated treatment of the patients should be monitored at least every 3 months, until the disease is stable (Table 1).

To evaluate colchicine response; a consensus was reached for the following aspects: decrease in duration and number of attacks is confirmative for defining response to colchicine treatment, colchicine resistance is defined as the presence of six or more attacks per year or $\geq 3$ attacks in a 4-6month period or elevation of two or more of the acute phase reactants in incomplete attacks, or evidence of subclinical inflammation between attacks (Table 1).

For biologic treatment; a consensus was reached for the following aspects: biologic agents should be commenced in resistant FMF patients and patients with amyloidosis, decrease in duration and number of attacks and cease of subclinical inflammation are accepted as response to biologics, prolongation of treatment intervals of biologic agents should be considered, in patients without any attack and laboratory evidence of subclinical inflammation within the last 6 months following initiation of biologics, the treatment intervals can be prolonged to twice the original dose intervals, after the prolongation of treatment intervals, in patients without any attack and laboratory evidence of subclinical inflammation within the last 1 year, the treatment intervals can be prolonged to three times the original dose intervals (Table 1).

\section{Indecisive statements}

Responses were indecisive (insufficient to form a consensus in neither direction), regarding the following aspects: initiating colchicine therapy to patients with a positive genetic testing with two pathogenic alleles but without the typical features of FMF, routine testing of serum amyloid A (SAA) or protein/creatinine in spot urine at each visit, formal scoring systems such as Auto-Inflammatory Diseases Activity Index (AIDAI), visual analogue scale (VAS) and FMF50 scoring as applicable at each visit, erythrocyte sedimentation rate (ESR) testing during an FMF attack, the use of formal scoring systems to evaluate the response to colchicine 
Table 1 The statements that reached a consensus with the median scores and the numbers of participants who agreed

Median (Mini- Number of parmum-maxi- ticipants who agreed mum)

1. Diagnosis of FMF

FMF should be diagnosed according to certain classification criteria

Genetic analysis should be ordered to all patients when clinical findings support FMF

$9(9-9)$

The result of genetic testing alone cannot be considered decisive for diagnosis

$9(2-9)$

2. Initiation of colchicine therapy

Colchicine is the first choice of treatment for FMF patients

$9(3-9)$

Colchicine therapy should be initiated at the time of diagnosis

$9(9-9)$

Colchicine therapy should be initiated when two pathogenic alleles and family history of amyloidosis

$9(9-9)$

are present, even in the absence of typical features

$9(2-9)$

3. Adjustment of colchicine dose

Colchicine therapy should be started at low doses $(0.5 \mathrm{mg} /$ day $)$ and dose should be increased until the maximum dose for the patient (based on the calculation depends on age/body surface area/body weight of the patient) (maximum $2 \mathrm{mg} /$ day) for receiving a response without emergence of side effects

Colchicine dose is calculated based on the age of the patients in routine clinical practice $(\leq 0.5 \mathrm{mg}$ for $<5$ years of age, $1 \mathrm{mg}$ for $5-10$ years of age, $1.5 \mathrm{mg}$ for $>10$ years of age)

Laboratory evidence of subclinical inflammation is an indication for increasing colchicine dose

Colchicine dose should be reduced if the liver enzymes were 2 times higher than normal levels

4. Screening periods

Screening periods of patients with FMF should not be longer than 6 months of duration

The newly initiated treatment of the patients should be monitored at least every 3 months, until the disease is stable

Colchicine-resistant patients should be monitored at least every 3 months

5. Evaluation of laboratory parameters

Complete blood count testing should be performed at each visit

CRP testing should be performed at each visit

ESR testing should be performed at each visit

SAA testing should be performed at each visit in patients resistant to colchicine

SAA testing should be performed at least once per year nevertheless the disease is stable

SAA testing should be performed in patients with elevation in other acute phase reactants

SAA testing should be performed in patients with a positive family history of amyloidosis

Liver and kidney function tests should be performed at each visit

Urinalysis should be performed at each visit

If proteinuria is detected in the urinalysis, it should be confirmed with quantitative tests in 24-h urine

6. Evaluation of FMF attacks

During an FMF attack, complete blood count testing should be performed

During an FMF attack, CRP testing should be performed

During an FMF attack, urinalysis should be performed to exclude other causes which would mimic an attack

7. Evaluation of response to colchicine

Decrease in number of attacks is indicative of a response to colchicine treatment

Decrease in duration of attacks is indicative of a response to colchicine treatment

Cease in subclinical inflammation is indicative of a response to colchicine treatment

8. Definition of colchicine resistance

Colchicine resistance is defined as the presence of six or more attacks per year or $\geq 3$ attacks in a 4-6 month period or elevation of two or more of the acute phase reactants in incomplete attacks, or evidence of subclinical inflammation between attacks

Failure to achieve an adequate improvement in quality of life scales is indicative for colchicine resistance 
Table 1 (continued)

\begin{tabular}{|c|c|c|}
\hline & $\begin{array}{l}\text { Median (Mini- } \\
\text { mum-maxi- } \\
\text { mum) }\end{array}$ & $\begin{array}{l}\text { Number of par- } \\
\text { ticipants who agreed } \\
(n)\end{array}$ \\
\hline \multicolumn{3}{|l|}{ 9. Commencing biologic treatment } \\
\hline Biologic agents should be commenced in colchicine resistant FMF patients & $9(7-9)$ & 14 \\
\hline Biologic agents should be commenced in patients with amyloidosis & $9(4-9)$ & 13 \\
\hline Colchicine treatment should not be discontinued in patients ongoing biological therapy & $9(7-9)$ & 14 \\
\hline \multicolumn{3}{|l|}{ Factors effecting the choice of biologic agents are } \\
\hline Cost & $7(2-9)$ & 11 \\
\hline Efficiency & $9(9-9)$ & 14 \\
\hline Ease of use & $9(7-9)$ & 14 \\
\hline Treatment adherence & $9(7-9)$ & 14 \\
\hline Accessibility & $8.5(7-9)$ & 14 \\
\hline Presence of adverse events during biologic agent treatment & $9(6-9)$ & 13 \\
\hline \multicolumn{3}{|l|}{ 10. Evaluation of response to biologic treatment } \\
\hline Decrease in number of attacks is indicative of a response to biologic treatment & $9(5-9)$ & 13 \\
\hline Decrease in duration of attacks is indicative of a response to biologic treatment & $8.5(5-9)$ & 13 \\
\hline Cease in subclinical inflammation is indicative of a response to biologic treatment & $9(6-9)$ & 13 \\
\hline $\begin{array}{l}\text { Outcome measurements such as AIDAI, VAS and FMF50 scoring should be performed at each visit to } \\
\text { evaluate response to biologics }\end{array}$ & $7(4-9)$ & 11 \\
\hline \multicolumn{3}{|l|}{ 11. Prolongation of treatment intervals of biologics } \\
\hline $\begin{array}{l}\text { In patients whose attacks are in remission under biologics, prolongation of treatment intervals of bio- } \\
\text { logic agents should be considered }\end{array}$ & $9(3-9)$ & 12 \\
\hline $\begin{array}{l}\text { Patients without any attacks and laboratory evidence of subclinical inflammation within the last } \\
6 \text { months following initiation of biologics, treatment intervals can be prolonged to twice the original } \\
\text { dose intervals }\end{array}$ & $7(2-9)$ & 11 \\
\hline $\begin{array}{l}\text { After the prolongation of treatment intervals, patients without any attacks and laboratory evidence of } \\
\text { subclinical inflammation within the last } 1 \text { year, treatment intervals can be prolonged to three times } \\
\text { the original dose intervals }\end{array}$ & $7.5(1-9)$ & 13 \\
\hline
\end{tabular}

$A I D A I$ auto-inflammatory diseases activity index, CRP C-reactive protein, ESR erythrocyte sedimentation rate, $F M F$ familial Mediterranean fever, $S A A$ serum amyloid A, VAS visual analogue scale

treatment, accepting adverse reactions to colchicine as a basis for initiating biologics.

\section{Discussion}

This study revealed the current real-life trends in the diagnosis and management of FMF from a country where the disease is highly prevalent. Since the survey was assessed by clinicians dealing with a large population of FMF patients, the results suggest a practical approach. Diagnosis and treatment patterns of the panellists mostly followed the current guidelines. As for the controversial issues of the literature such as evaluation of response to colchicine, or laboratory tests in the management of patients, a consensus was reached on many statements.

The diagnosis of FMF is based on the use of diagnostic criteria with the support of genetic testing. The initial implementation of genetic testing has brought a new perspective to diagnostic practices of FMF, and genetic testing is worthwhile both as a diagnostic adjunct and for predicting the severity of the disease and the patients' predispositions to comorbidities, since some mutations were found to be associated with a severe disease course in various studies [20-24].

Whether treatment should be commenced to asymptomatic patients with a positive genetic testing remains controversial in the literature. Various studies have indicated that environmental factors, type of the mutation in $M E F V$ gene and genetic changes other than $M E F V$ gene may be involve in the development of amyloidosis [25-27]. Current guidelines recommend following these patients closely and considering initiation of treatment if there are risk factors for amyloidosis such as country of residence, family history of amyloidosis and persistently elevated acute phase reactants $[12,14]$. The percentage of patients with amyloidosis and severity of the disease have been decreasing in Turkey [28, 29], therefore residing in Turkey alone was not considered to be associated with an increased risk of amyloidosis in asymptomatic patients by the panellists, and a consensus 
could not be reached in neither direction on initiation of colchicine treatment in asymptomatic patients, but it seems necessary to follow these patients closely. However, presence of a positive family history for amyloidosis is considered to be an indication to start colchicine in asymptomatic patients with two pathogenic alleles due to the possibility of additional genetic factors that may influence the development of amyloidosis.

With respect to colchicine dose adjustment, the evidence for optimum colchicine dose to prevent attacks in children is scarce. Our study revealed that in routine practice colchicine dose is adjusted mostly according to the patient's age. However, especially for young children, dose adjustment based on body surface area or body weight would be more effective. A recent study also revealed that the best correlation of colchicine intake with a positive response was calculating the dose according to body surface area [30, 31]. The dose can be increased up to $2 \mathrm{mg}$ for receiving a response without emergence of side effects.

During routine follow-up various laboratory tests are beneficial to evaluate drug effectiveness, side effects and subclinical inflammation; however, the evidence supporting monitorization of FMF with any acute phase reactant over the others is still limited [32]. The panellists prefer CRP and ESR testing in every visit due to their availability, but SAA testing at least once a year to every patient, and at each visit in patients who are resistant to colchicine, having other evidence of subclinical inflammation and having a family history of amyloidosis considering its role in the pathogenesis of AA amyloidosis [33].

White blood cell count and CRP testing were considered convenient to evaluate acute phase response during an attack by the panellists. In a study evaluating the laboratory parameters of 168 FMF patients, a correlation between CRP and SAA was found during FMF attacks, therefore the authors concluded that checking for SAA during an FMF attack is not required [33].

The rate of colchicine resistance is reported as 5-10\%, and partially response rate is reported as $30-40 \%$ in the previous studies [34, 35]. Colchicine resistance was reported $5-15 \%$ by $92.86 \%$ of our panellists similar to the literature, but $7.14 \%$ of the panellists reported higher rates. There is no universally accepted tool for evaluating the response to colchicine and determining colchicine resistance in the literature. Ben-Chetrit et al. proposed to use a scoring system based on percentage reduction in number of attacks [36]. Hentgen et al. defined colchicine resistance as suffering from either more than six typical FMF attacks per year or more than three typical FMF attacks within 4-6 months. In case of incomplete attacks, an increase in at least two out of three acute phase reactants (CRP, ESR and SAA) between attacks is considered mandatory for defining a patient as unresponsive to colchicine [35]. In the recent EULAR recommendations colchicine resistance is defined as having one or more attacks each month despite receiving the maximally tolerated dose of colchicine for at least 6 months [14]. The panellists defined colchicine resistance as the presence of six or more attacks per year or $\geq 3$ attacks in a 4-6 month period or elevation of two or more of the acute phase reactants in incomplete attacks, or evidence of subclinical inflammation between attacks, or failure to achieve an adequate improvement in quality of life scales or less than $50 \%$ reduction in FMF 50 scoring [37] for 5 out of 6 criteria.

Favourable results have been reported for anti-IL-1 treatments and it has become first choice in patients resistant to colchicine therapy although its long term efficacy and safety has not been yet clarified [38-44]. The panellists accepted the colchicine resistance as an indication for the anti-IL-1 therapy and they reported that they continue colchicine on anti-IL-1 therapy, it's also recommended in the current guidelines as colchicine is the only proven medication in preventing secondary amyloidosis $[14,35]$.

One of the most noticeable results of the study was the consensus among the panellists that, after a while, treatment intervals could be extended. This opinion was based on the clinical observation that some patients who discontinued anti IL-1 treatment did not experience an attack for a long time, even though they had frequent attacks before initiation of biologic treatment. This could be explained by resetting the autonomous inflammatory state with IL-1 blocking [45]. The protocol for prolonging the biologic treatment intervals was solely determined subjectively by the participants and is needed to be confirmed by controlled studies.

Owing to its interactive, iterative and systematic nature, we believed that the Delphi technique provided more clarified data on controversial aspects compared to a conventional survey. However, our study has some limitations. All participants come from the same country and the number of the participants are limited. Turkey is a country where FMF is highly prevalent and all paediatric rheumatologists in Turkey deal with a large number of FMF patients in their routine clinical practice. The fact that all participants are coming from Turkey may be considered as a limitation, but this might also have led to more uniform results. An international study is also in the future plans of the authors. Another limitation of the study was the lack of a consensus on some aspects as reported in the literature. We believe a stronger scientific evidence is needed on these aspects to form a uniform agreement.

In conclusion, in this study a consensus was achieved regarding the routine screening periods of FMF patients with the convenient laboratory parameters. Recommendations for the initiation of colchicine treatment in asymptomatic patients were made and adjustment of colchicine treatment dose in routine practise was revealed. The definition of colchicine resistance was made and a protocol was created for 
prolongation of treatment intervals of biologic agents. We anticipate that the results of the study reveal real-life data on the approach to patients in clinical practice. An international survey may enable us to reach an agreement on issues that we did not reach a consensus.

\section{Acknowledgements None.}

Author contributions Conceptualization: BS, NAA. Design of the work: NAA, BS, GKK, HES. Data analysis: NAA, BS, HES, GKK. All authors made substantial contributions to the interpretation of data for the work. All authors were involved in drafting the article or revising it critically, and all authors approved the final version of the manuscript. All authors are accountable for all aspects of the work and will ensure questions related to the accuracy or integrity of any part of the work will be appropriately investigated and resolved.

Funding No specific funding was received from any bodies in the public, commercial or not-for profit sectors to carry out the work described in this article.

Data availability The data underlying this article will be shared on reasonable request to the corresponding author.

\section{Compliance with ethical standards}

Conflict of interest The authors declare no conflicts of interest.

Ethical approval Approval was obtained for the study protocol from the Ethics Committee of Istanbul University, Istanbul Faculty of Medicine (approved: 31/08/2020-145479). The study was carried out complied with the Declaration of Helsinki.

\section{References}

1. Ben-Chetrit E, Touitou I (2009) Familial mediterranean Fever in the world. Arthritis Rheum 61(10):1447-1453

2. Ozen S, Bilginer Y (2014) A clinical guide to autoinflammatory diseases: familial Mediterranean fever and next-of-kin. Nat Rev Rheumatol 10(3):135-147

3. Gül A (2014) Treatment of familial Mediterranean fever: colchicine and beyond. Isr Med Assoc J 16(5):281-284

4. Meinzer U, Quartier P, Alexandra JF, Hentgen V, Retornaz F, Koné-Paut I (2011) Interleukin-1 targeting drugs in familial Mediterranean fever: a case series and a review of the literature. Semin Arthritis Rheum 41(2):265-271

5. Ben-Zvi I, Kukuy O, Giat E, Pras E, Feld O, Kivity S et al (2017) Anakinra for colchicine-resistant familial Mediterranean fever: a randomized, double-blind. Placebo-Controlled Trial Arthritis Rheumatol 69(4):854-862

6. Özen S, Batu ED, Demir S (2017) Familial Mediterranean fever: recent developments in pathogenesis and new recommendations for management. Front Immunol 8:253

7. Livneh A, Langevitz P, Zemer D, Zaks N, Kees S, Lidar T et al (1997) Criteria for the diagnosis of familial Mediterranean fever. Arthritis Rheum 40(10):1879-1885

8. Yalçinkaya F, Ozen S, Ozçakar ZB, Aktay N, Cakar N, Düzova A et al (2009) A new set of criteria for the diagnosis of familial Mediterranean fever in childhood. Rheumatology (Oxford) 48(4):395-398

9. Kondi A, Hentgen V, Piram M, Letierce A, Guillaume-Czitrom S, Koné-Paut I (2010) Validation of the new paediatric criteria for the diagnosis of familial Mediterranean fever: data from a mixed population of 100 children from the French reference centre for auto-inflammatory disorders. Rheumatology (Oxford) 49(11):2200-2203

10. Demirkaya E, Saglam C, Turker T, Koné-Paut I, Woo P, Doglio $M$ et al (2016) Performance of different diagnostic criteria for familial Mediterranean fever in children with periodic fevers: results from a multicenter international registry. J Rheumatol 43(1):154-160

11. Gattorno M, Hofer M, Federici S, Vanoni F, Bovis F, Aksentijevich I et al (2019) Classification criteria for autoinflammatory recurrent fevers. Ann Rheum Dis 78(8):1025-1032

12. Giancane G, Ter Haar NM, Wulffraat N, Vastert SJ, Barron K, Hentgen $\mathrm{V}$ et al (2015) Evidence-based recommendations for genetic diagnosis of familial Mediterranean fever. Ann Rheum Dis 74(4):635-641

13. Gül A (2016) Approach to the patients with inadequate response to colchicine in familial Mediterranean fever. Best Pract Res Clin Rheumatol 30(2):296-303

14. Ozen S, Demirkaya E, Erer B, Livneh A, Ben-Chetrit E, Giancane $G$ et al (2016) EULAR recommendations for the management of familial Mediterranean fever. Ann Rheum Dis 75(4):644-651

15. Tunca M, Akar S, Onen F, Ozdogan H, Kasapcopur O, Yalcinkaya $\mathrm{F}$ et al (2005) Familial Mediterranean fever (FMF) in Turkey: results of a nationwide multicenter study. Medicine (Baltimore) 84(1):1-11

16. Sozeri B, Sönmez HE, Demir F, Çakan M, Öztürk K, Özdel S, et al. Time to collaborate: objectives, design, and methodology of PeRA-Research Group. Northern Clinics of İstanbul

17. Turoff M, Linstone, H. A. Introduction. In: Turoff M, Linstone, H. A., editor. The Delphi method-techniques and applications 2002. p. 3-12

18. Diamond IR, Grant RC, Feldman BM, Pencharz PB, Ling SC, Moore AM et al (2014) Defining consensus: a systematic review recommends methodologic criteria for reporting of Delphi studies. J Clin Epidemiol 67(4):401-409

19. Ruperto N, Meiorin S, Iusan SM, Ravelli A, Pistorio A, Martini A (2008) Consensus procedures and their role in pediatric rheumatology. Curr Rheumatol Rep 10(2):142-146

20. Ben-Chetrit E, Backenroth R (2001) Amyloidosis induced, end stage renal disease in patients with familial Mediterranean fever is highly associated with point mutations in the MEFV gene. Ann Rheum Dis 60(2):146-149

21. Papa R, Doglio M, Lachmann HJ, Ozen S, Frenkel J, Simon A et al (2017) A web-based collection of genotype-phenotype associations in hereditary recurrent fevers from the Eurofever registry. Orphanet J Rare Dis 12(1):167

22. Ayaz NA, Tanatar A, Karadă Ş, Çakan M, Keskindemirci G, Sönmez HE (2020) Comorbidities and phenotype-genotype correlation in children with familial Mediterranean fever. Rheumatol Int

23. Barut K, Sahin S, Adrovic A, Sinoplu AB, Yucel G, Pamuk G et al (2018) Familial Mediterranean fever in childhood: a singlecenter experience. Rheumatol Int 38(1):67-74

24. Ozcakar ZB, Cakar N, Uncu N, Celikel BA, Yalcinkaya F (2017) Familial Mediterranean fever-associated diseases in children. QJM 110(5):287-290

25. Ozen S, Demirkaya E, Amaryan G, Kone-Paut I, Polat A, Woo $P$ et al (2014) Results from a multicentre international registry of familial Mediterranean fever: impact of environment on the expression of a monogenic disease in children. Ann Rheum Dis 73(4):662-667

26. Akpolat T, Ozkaya O, Ozen S (2012) Homozygous M694V as a risk factor for amyloidosis in Turkish FMF patients. Gene 492(1):285-289 
27. Gershoni-Baruch R, Brik R, Zacks N, Shinawi M, Lidar M, Livneh A (2003) The contribution of genotypes at the MEFV and SAA1 loci to amyloidosis and disease severity in patients with familial Mediterranean fever. Arthritis Rheum 48(4):1149-1155

28. Akse-Onal V, Sağ E, Ozen S, Bakkaloglu A, Cakar N, Besbas N et al (2010) Decrease in the rate of secondary amyloidosis in Turkish children with FMF: are we doing better? Eur J Pediatr 169(8):971-974

29. Kisla Ekinci RM, Balci S, Dogruel D, Altintas DU, Yilmaz M (2019) Twenty-Year experience of a single referral center on pediatric familial Mediterranean fever: what has changed over the Last decade? J Clin Rheumatol

30. Kallinich T, Haffner D, Niehues T, Huss K, Lainka E, Neudorf $\mathrm{U}$ et al (2007) Colchicine use in children and adolescents with familial Mediterranean fever: literature review and consensus statement. Pediatrics 119(2):e474-e483

31. Knieper AM, Klotsche J, Lainka E, Berger T, Dressler F, Jansson AF et al (2017) Familial Mediterranean fever in children and adolescents: factors for colchicine dosage and predicting parameters for dose increase. Rheumatology (Oxford) 56(9):1597-1606

32. Erer B, Demirkaya E, Ozen S, Kallinich T (2016) What is the best acute phase reactant for familial Mediterranean fever followup and its role in the prediction of complications? A systematic review. Rheumatol Int 36(4):483-487

33. Çakan M, Karadağ Ş, Tanatar A, Sönmez HE, Ayaz NA (2019) The value of serum amyloid a levels in familial Mediterranean fever to identify occult inflammation during asymptomatic periods. J Clin Rheumatol

34. Ozen S, Kone-Paut I, Gul A (2017) Colchicine resistance and intolerance in familial Mediterranean fever: definition, causes, and alternative treatments. Semin Arthritis Rheum 47(1):115-120

35. Hentgen V, Grateau G, Kone-Paut I, Livneh A, Padeh S, Rozenbaum $\mathrm{M}$ et al (2013) Evidence-based recommendations for the practical management of Familial Mediterranean Fever. Semin Arthritis Rheum 43(3):387-391

36. Ben-Chetrit E, Ozdogan H (2008) Non-response to colchicine in FMF-definition, causes and suggested solutions. Clin Exp Rheumatol 26(4 Suppl 50):S49-51
37. Ozen S, Demirkaya E, Duzova A, Erdogan O, Erken E, Gul A et al (2014) FMF50: a score for assessing outcome in familial Mediterranean fever. Ann Rheum Dis 73(5):897-901

38. Kuijk LM, Govers AM, Frenkel J, Hofhuis WJ (2007) Effective treatment of a colchicine-resistant familial Mediterranean fever patient with anakinra. Ann Rheum Dis 66(11):1545-1546

39. Rossi-Semerano L, Fautrel B, Wendling D, Hachulla E, Galeotti C, Semerano L et al (2015) Tolerance and efficacy of off-label anti-interleukin-1 treatments in France: a nationwide survey. Orphanet J Rare Dis 10:19

40. Laskari K, Boura P, Dalekos GN, Garyfallos A, Karokis D, Pikazis D et al (2017) Longterm beneficial effect of canakinumab in colchicine-resistant familial Mediterranean fever. J Rheumatol 44(1):102-109

41. Gül A, Ozdogan H, Erer B, Ugurlu S, Kasapcopur O, Davis N et al (2015) Efficacy and safety of canakinumab in adolescents and adults with colchicine-resistant familial Mediterranean fever. Arthritis Res Ther 17:243

42. Hentgen V, Vinit C, Fayand A, Georgin-Lavialle S (2020) The use of interleukine-1 inhibitors in familial Mediterranean fever patients: a narrative review. Front Immunol 11:971

43. Ozen S, Ben-Cherit E, Foeldvari I, Amarilyo G, Ozdogan H, Vanderschueren $\mathrm{S}$ et al (2020) Long-term efficacy and safety of canakinumab in patients with colchicine-resistant familial Mediterranean fever: results from the randomised phase III CLUSTER trial. Ann Rheum Dis 79(10):1362-1369

44. De Benedetti F, Gattorno M, Anton J, Ben-Chetrit E, Frenkel J, Hoffman HM et al (2018) Canakinumab for the treatment of autoinflammatory recurrent fever syndromes. N Engl J Med 378(20):1908-1919

45. Gül A (2018) Dynamics of inflammatory response in autoinflammatory disorders: autonomous and hyperinflammatory states. Front Immunol 9:2422

Publisher's Note Springer Nature remains neutral with regard to jurisdictional claims in published maps and institutional affiliations.

\section{Affiliations}

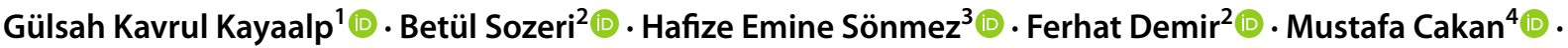

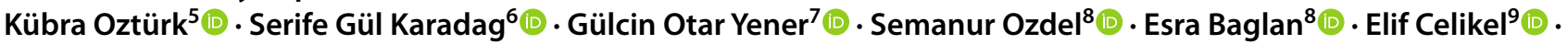

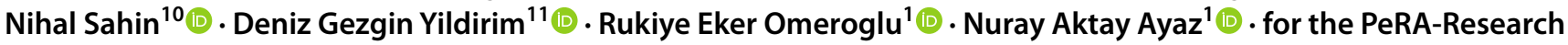 Group}

1 Department of Paediatric Rheumatology, Istanbul University Faculty of Medicine, Fatih, Istanbul, Turkey

2 Department of Paediatric Rheumatology, University of Health Sciences Umraniye Research and Training Hospital, Istanbul, Turkey

3 Department of Paediatric Rheumatology, Kocaeli University Faculty of Medicine, Kocaeli, Turkey

4 Department of Paediatric Rheumatology, Zeynep Kamil Research and Training Hospital, Istanbul, Turkey

5 Department of Paediatric Rheumatology, Istanbul Medeniyet University Göztepe Research and Training Hospital, Istanbul, Turkey

6 Department of Paediatric Rheumatology, Istanbul Bakırköy Dr. Sadi Konuk Research and Training Hospital, Istanbul, Turkey
7 Department of Paediatric Rheumatology, Sanliurfa Research and Training Hospital, Sanliurfa, Turkey

8 Department of Paediatric Rheumatology, University of Health Sciences Ankara Dr. Sami Ulus Research and Training Hospital, Ankara, Turkey

9 Department of Paediatric Rheumatology, Adana City Research and Training Hospital, Adana, Turkey

10 Department of Paediatric Rheumatology, Bursa City Hospital, Bursa, Turkey

11 Department of Paediatric Rheumatology, Diyarbakır Children's Hospital, Diyarbakir, Turkey 